\title{
LE JUIF NGAI, INFORMATEUR DU P. MATHIEU RICCI,
}

PAR

\section{PAUL PELLIOT.}

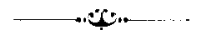

Dès 1615 , la version latine publiée par Trigault des Commentaires de Mathieu Ricci racontait comment, quelques années après l'établissement des Jésuites à Pékin, un Juif de K'ai-fong-fou était venu trouver le P. Ricci qu'il prenait pour un correligionnaire, et avait ainsi rérélé à l'Occident l'existence ancienue de colonies juires en Extrême-Orient.

Il y a quelques années, l'édition da P. Tacchi-Venturi nous a rendu les Commentaires de Ricci dans le texte italien original ${ }^{1}$ ). Aux pages 468-471, sous une date que le P. Tacchi-Venturi fixe à 1605 , on retrouve le récit traduit par Trigault. Il vient d'être question d'un livre écrit par un Chinois et où il était question des Pères, et Ricci continue ainsi:

"Ce livre vint aux mains d'un Juif de nation et de religion, de la province et de la métropole du Ho-nan, NgaI de son nom

1) P. Pietro Tacchi-Venturi S.I., Opere Storiche del P. Matteo Ricci S.J., t. I, I Commentari della China, Macerata, 1911, grand in $-8^{\circ}$, cxvil +650 pp. +1 f. s. n.; t. II, Le lettere della Cina, ibid., 1913, Lxvi +570 pp. $+i$ f. s. n., avec planches. L'édition est excellente et le commentaire met à profit les précieuses archives de la Compagnie. Un certain nombre de mots chinois sont estropiés. L'index est très incomplet et assez souvent fautif. 
de famille, lequel avait déjà atteint le grade de licencié littéraire en Chine et était venu cette même année à Pékin pour les examens de doctorat.... Le P. Matbieu le mena de suite à la chapelle ò̀ on avait nouvellement placé sur l'autel, vu que c'était la fête de Saint Jean Baptiste, une belle image de la Vierge, qui avait d'un côté l'Enfant Jésus, et de l'autre Saint Jean Baptiste en adoration à genoux.... On lui montra la Bible de Plantin en hébreu.... Il dit aux pères qu'à K'ai-fong beaucoup savaient l'hébreu, entre autres un sien frère, et que Jui, dès son enfance, s'était adonné aux lettres chinoises, et ainsi n'avait pas appris les lettres hébraïques. Et il donna à entendre que, pour avoir suivi les choses des lettrés de la Chine, il avait été chassé de la synagogue par l'archiprêtre qui est là à leur tête et était à moitié excommunié, et qu'il aurait facilement abandouné cette loi s'il eût pu obtenir le grade de docteur, comme le font aussi les Musulmans qui, réussissant à obtenir le grade de docteur, n'ont plus peur de leurs mollah, et abandonnent la loi ${ }^{1}$ ).

Ainsi le licencié Ngai serait venu voir Ricci le jour de la SaintJeau, e'est-à-dire le 24 juin 1605, à l'occasion d'un royage que Ngai faisait à Pékin pour se présenter aux examens triennaux de doctorat. On sait en effet que ces examens triennaux ont amené au P. Ricei, tout au moins en 1607 et 1610, un grand concours de visiteurs.

Mais il y a à cette version des Commentaires une grosse difficulté. Les dates des examens triennaux de doctorat sont bien connues. Depuis le début des Ming, les noms des lauréats de chaque promotion sont gravés sur des stèles qui existent encore au Kouo-tseu-kien de Pékin, et toutes ces promotions sont éditées dans un recueil

1) Ngai exagère l'opposition que les rabbins auraient faite aux lettres chinoises. Les Inscriptions juives de $K^{\prime}$ ai fong fou traduites par le P. Tobar font conuaîlre les noms de plusieurs bacheliers et même de delıx docteurs, Kao Sitan (de promotion inconnue) et Tchao Ying-cheng, docteur de 1646, qui conservèrent des attaches étroites avec la synagogue. 
facilement accessible, le 題名碑鈛 $T$ $i$ ming pei lou. Or il y a eu des examens de doctorat en 1601, en 1604, en 1607; il n'y en a pas eu en 1605 .

On ne pourrait d'ailleurs arguer de l'absence de millésime précis dans ce chapitre des Commentaires pour reporter à juin 1604 la visite du Juif Ngai. Les examens de doctorat se passaient au printemps, et l'Histoire des Ming enregistre régulièrement, dans la troisième lune, la proclamation des résultats; en 1604, c'est le 13 avril que la liste sortit ${ }^{1}$ ). Peu après, les candidats malheureux retournaient dans leurs provinces. Il n'y aurait pas grande apparence pour que le Juif Ngai, s’il était venu à Pékin pour les examens de doctorat, s'y fût encore trouvé le 24 juin. D'ailleurs les Commentaires disent que, lors de la visite de Ngai, on lui montra la Bible de Plantin en hébreu. Il s'agit là de la Bible tétraglotte dite «Bible Royale», imprimée chez Plantin en huit volumes, et qui parvint à Pékin, sauvée d'un naufrage dans une inondation du Pei-ho aux environs de T'ong-tcheou, au plus tôt dans les derniers jours de juillet $1604^{2}$ ): même à cette date, la Saint-Jean était passée depuis un mois. Enfin il y a une raisou décisive pour que la visite de Ngai soit bien de 1605 ; c'est que dans une lettre autographe du 26 juillet 1605, adressée au P. Cl. Acquaviva, le P. Ricci emploie en propres termes, à propos de la visite de Ngai, l'expression de «ces jours passés».

C'est en reprenant cette lettre du 26 juillet 1605 que nous allons

1) Cf. Hing che, ch. $21, f^{\circ} 2 \mathrm{r}^{\circ}$.

2) Sur la venue de cette Bible, cf. Tacchi-Venturi, I, 453; II, 260, 282-283. Le P. Tacchi-Venturi $(I, 452)$ admet mème que le naufrage (dont il est aussi question II, 266) est de l'automne ou de l'hiver de 1604. I'hiver est hors de question, puisque le Pei-ho est alors gelé et ne peut déborder. En outre le P. Ricei (cf. Tacchi-Venturi, I, 452) met ce naufrage et l'inondation du Pei-ho au même temps que les grandes piluies qui cansèrent une inondation à Pékin (sar ces plujes, cf. Tacchi-Venturi, I, 452; II, 271, 288). Or, d'après le Ming che (ck. 21, fo $3 \mathrm{r}^{\circ}$ ), c'est le 27 juillet que «à la capitale il $\mathbf{y}$ eut une grande pluie qui renversa [une partie de] l'enceinte de la ville». 
chercher la solution de la difficulté. Le P. Ricci dit qu'il a appris depuis "peu de jours» l'existence d'une ancienue chrétienté à K'ai-fong-fou, et il continue en ces termes (Tacchi-Venturi, II, 290 et suiv.):

«Nous arons sti cela par l'intermédiaire d'un Juif de religion, de nationalité et de type, qui ces jours passés est venu me visiter... C'est un homme appelé Ngai, de la province du Ho-nan, habitant de la métropole [de cette province]; son père avait trois fils; Iui s'est adonné aux lettres chinoises et a obtenu le grade de licencié; il a déjà une soixantaine d'années; il est venu cette année demander un emploi qui lui a été donué dans une école de la ville de Ianceo (Yang-tcheou). Les deux autres frères se sout appliqués aux lettres hébraïques et sont, paraît-il, rabbins parmi eux ${ }^{1}$ ).... Il viut chez nous dans l'octave de Saint Jean Baptiste....»

Cette lettre, écrite au lendemain de la visite de Ngai, doit faire foi. Avec elle, toute difficulté disparait. Ngai vint en 1605 à Pékin pour demander un emploi, et non pour passer un examen qui n'eut pas lieu cette année-là. Mais quelques aunées plus tard, en écrivant ses Commentaires, Ricci confondit la visite de ce licencié avec celles que lui rendirent tant d'autres licenciés quand ils venaient à Pékin pour les examens triennaux de doctorat. Il est possible d'ailleurs que Ngai ait dit aux Pères, comme le veulent les Commentaires, qu'il se fut senti plus libre d'abandonner ses correligionnaires s'il eût pu passer le doctorat ${ }^{2}$ ); mais à 60 aus, il avait eu le temps d'échouer à pas mal de sessions, et en 1605 ce n'est pas pour tenter encore de conquérir un grade littéraire qu’il était venu à la capitale, mais pour obtenir un gagne-pain. Enfin la date de cette

1) Deux ou trois ans plus tard, le fils d'un de ces frères rabbins, appelé lui aussi Ngai naturellement, vint à son tour visiter les Jésuites de Pékin.

2) Des propos prêtés à Ngai, il semble résulter que Juifs et Musulmans constituaient en Chine sous les Ming, au point de vue administratif, des «nations" placées sous l'autorité et la responsabilité de leurs chefs religieux. 
visite n'est pas le 24 juin, mais un jour indéterniné de la semaine du 25 au 31 juiu 1605.

Ce Juif Ngai, il n'est pas inıpossible de l'identifier.

Il $y$ a peu de noms de famille Ngai; celui auquel on songe immédiatement est celui-là même que prit peu après le P. Aleni, à savoir 苂 Ngai. La Biographie chinoise de Ricci écrite par le P. Aleni ${ }^{1}$ ), en racontant la visite de 1605 , donne bien au Juif le

1) Il n'y a à ma connaissance (si on excepte la portion dn ch. 326 da Ming che partiellement et parfois inexactement traduite dans Bretschneider, Mediaval Researches, II, 32s-326) qu'une biographie chinoise du P. Ricci, celle dâe au P. Aleni. Elle est précédée d'un portrait du P. Ricci qui a été reproduit par le P. Tacchi-Venturi (t. II, en face de la p. 172) d'après l'exemplaire imprimé de cette Biographie, en $20 \mathrm{ff}$. plus ] fo de frontispice, qui se troure à la Bibliothèque Nationale de Rome. M. Vacea (duns Tacchi-Venturi, II, 548), qui mentionne cet exemplaire imprimé de Rome (sous le $n^{\circ} 2 \mathbf{i}$ de sa bibliographie), ne donne aucune date soit pour la rédaction, soit pour l'impression; mais il ajoute qu'il y a également ì la Bibliothèque Nationale de Rome un exemplaire imprimé de deux autres «biographies" (qu'il classe sous les $\mathrm{n}^{\text {o* }} 22$ et 23 de sa bibliographie), l'une en 10 ff., l'autre en 2 ff. Pour aucune de ces deux autres «biographies», il n'indique de nom d'auteur, ni de date de composition ou d'impression. Mais M. Vacea dit que son $n^{\circ} 21$ correspond au $\mathrm{L}^{\circ} 28$ de Cordier, L'imprimerie sino-europrenne en Chine, et ses $\mathrm{n}^{\prime \prime \prime} 22$ et 23 au $\mathrm{n}^{\circ} 29$ du même onvrage de $M$. Cordier. Vu que, pour le $\mathrm{n}^{\circ} 28$, M. Cordier indique une postface qu'il date de 1636, je pense que c'est là que le P. 'lacchi-Venturi a pris cette même date pour le portrait de Ricci. En réalité, il y a eu dans les divers auteurs toute une série de confusions. Il faut renverser les équivalences données par M. Vacca; c'est le $\mathrm{n}^{\circ} 29$ de $\mathbf{M}$. Cordier qui répond à son $n^{\circ} 21$, et le $n^{\circ} 2 S$ de $M$. Cordier représente en réalité ses $n^{\circ s}$ 22 et 23, plus autre chose. Mais les descriptions mêmes de M. Cordier reproduisent à leur tour des indicutions en partie inexactes dn Catalogue des livres chinois de M. Courant. Les biographies chinoises du P. Ricci, toutes manuserites, occupent dans ce Catalogue les $\mathrm{n}^{\mathrm{ng}} 1014,1015,1016$ et 996. Les $\mathrm{n}^{\mathrm{j} *}$ 1014, 1015 et 1016 contiennent la Biographie de Ricci par Aleni. Le $\mathrm{n}^{\circ} 1014$ en partienlier est la reproduction fidèle d'un exemplaire imprimé à l'église de Fon-tcheou, appeléc ici 积 教 点 King-kiao-teang; il est indiqué que l'ueure a été relue par les PP. Emmanuel Uiaz (junior), Fereira et Semedo, et que l'édition a été autorisée par le P. Emmanuel Diaz (junior), supérieur de la Mission. Il est vraisemblable que c'est là l'élition même qui existe à la Bibliothèque Nationale de Rome, encore qu'il ait dû y avoir aussi au XVII" siècle une édition publiée par la mission de Pékin (à en juger d'après les listes de Conrant, Catal., n 7046). Je doute d'ailleurs que, pour l'édition de Pékin, il faille adopter la date de 1620 indiquée par le P. Sommervogel, car il me jaraît probable que la révision des PP. Ĺm. Diaz, Fereira et Semedo et l'autorisation du P. Diaz sappliquent à la première édition; or le P. Diaz (junior) n'est devenu vice-provincial que vers 1623 ; d'autre part la révision est forcément antérieure à 1637, 
nom de 茂 Ngai, et les inscriptions juives de $\mathrm{K}$ ai-fong-fou nous attestent que ce nom était effectivement porté par des familles de

date à laquelle le P. Semedo quitta la Chine. Enản, à supposer que l'édition de Fou-tcheou ne soit pas l'édition princeps, elle doit être antérieure à $164 \mathbf{5}$, date à laquelle l'église de Fou-tcheou cessa d'être appelée King-kiaot'ang. Comme on le voit, il n'y a rien là qui s'oppose à la date de 1636; mais cette date elle-mêne, jusqu'à de nouvelles trouvailles, ne me paraît reposer sur rien. Dans le $n^{\circ} 1015$, la biographie de Ricci par Aleni est suivic du rapport de 喿道居 Wou Tao-nan dernandant que l'Empereur octroie un terrain jour la sépulture de Rícci (Wou 'J'ao-nan avait été reçu pang-yen, e'est-à-dire sccond, atux

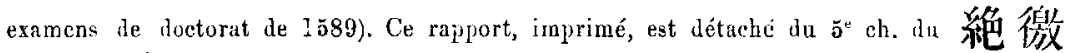
同文紟 Tsiue kiao toong wen ki, oì il occupait les fi. 10-13. Le Tsiue kiao t'ong wen ki, qui semble aujourd'hui perdu, doit avoir été, à en jugrer par son titre, un ouvrage assez considérable consacré à des documents coneernant des étrangers lointains, c'est-à-dire sans doute les missionnaires. J'en ai retrouvé la préface, écrite en 16] 5 par le mandarin chrétien 杵廷第 Iang Teing-yun, au debut du très intéressant recueil de documents relatifs aus chrétiens qui se trouve à la Bibliothèque publique de Petrograd sous le titre de $大$

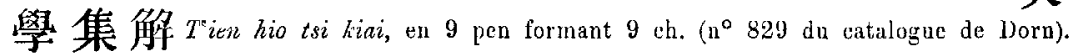
Eutin le ॥ $^{\circ} 996$ de M. Conrant, d'où la date de 1636 paraît tirée, ne la donne pas. Dans un examen forećment superticiel, M. Courant a indiqué inexactement le contenu du manuscrit. En tête de ce $n^{\circ} 996$ est le portrait de Ricci, copié sur celui de l'édition imprirnéc de la

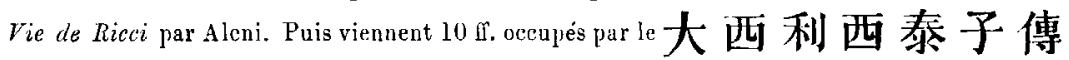
Ta si $l i$ si $t^{\circ}$ ai tseu tchouan (correspondant manifestement au $\mathrm{n}^{\circ} 22 \mathrm{~d}, \mathrm{M}$. Vucca), lequel

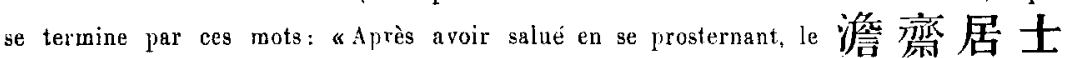

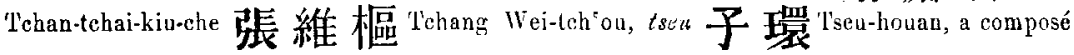
[cet écrit]». Tchang Wei-tch"on (de Wen-ling ou Tsin-kiang, c'est-à-dire de 'Ts'inan-tcheou au Fou-kien) était gouverneur du Chàn-si en 1626 (cf. Chàn si t'ong tche de 1735, ch. 22, $f^{\circ} 23 \mathrm{r}^{\circ}$ ); il est nommé dans un édit de 1628 qu'on retrouve dans les cuuves du ministre

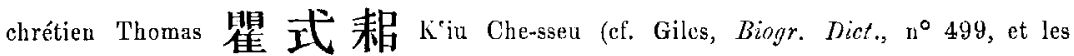
auvres de Kiu Che-sscu, à la Bibl. Nat, coll. Pelliot, II, 236, ch. 2, f० 24 $\mathrm{r}^{\circ}$ ); une poésie écrite en fuveur des missionnaires par Tchang Wei-tcheou antérieurement à 1641 se

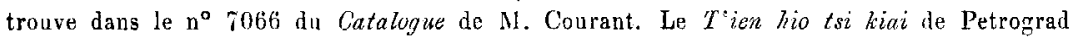
contient une dissertation de Tchang Wei-tch ou sur le Si hio fan et le Wan wou tohen yuan d'Aleni. Si j'avais actuellement à ma disposition le Tsiuan tcheou fou tohe, il est probable que je pourrais préeiser les étapes de la carrière de ce fonctionnaire lettré. En lout cas, sa biographie de Ricei, qui est sans doute antérieure à 1650, n'est qu’un démarquage de celle écrite par Aleni, qu’il invoque formellement. Vient ensuite, dans le manuscrit $n^{\circ} 996$, la biographie mêrne écrite par Aleni, avee la même suscription que dans les $\mathrm{n}^{0 *} 1014-1016$,

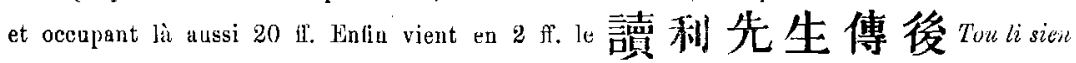

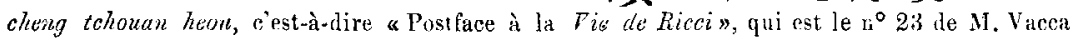

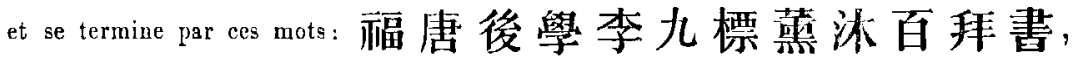
“Ecrit, aprc̀s s'être parfumé et lavé et avoir salué cent fois, par le disciple Li Kieon-piao, 
la colonie juive du Ho-nan ${ }^{\mathrm{I}}$ ). Mais ce nom même de 艾 Ngai est assez rare. D'autre part, les monographies locales enregistrent le plus sourent les promotions de licenciés pour les préfectures ou sous-préfectures dont elles s'occupent. Or, dans la Description de $K$ 'ai-fong-fou, il n'y a, pour les deux sous-préfectures de Siang-fou et de Siuan-wou qui se partagent la ville même de K'ai-fong, qu'un seul licencié de nom de famille Ngai, c'est 艾 田 Ngai T'ien, originaire de la sous-préfecture de Siaug-fou, qui passa l'examen de

de Fou-teang (Fou-tcheou)". Ii Kieou-piao et son frère 李 九为 Li Kieou-kong sont deux chrétiens connus; Li Kieou-kong, converti en 1628 (voir sa préface à Courant, Catalogue, $\mathrm{n}^{\circ}$ 6876), est mort en 1681; sur $\mathrm{Li}$ Kieou-jiao, on peut consulter les $\mathrm{n}^{\circ 8} 6884$ et 7114 du Catalogue de Courant, et Havret, Stèle chrétienne, Il, 95. L'activité littéraire des deux frères paraìt porter sur le $2^{e}$ tiers du XVII siècle. Entin, une dernière ligne porte: “Copié (金承) au $11^{*}$ mois de ping-tseu, 33 feuillets». C'est de ce ping-tseu que M. Courant a tiré la date de 1636 , qui a passé dans l'Imprimerie sino-européenne de $\mathbf{M}$. Cordier et de li, semble-t-il, dans l'ouvragre du P. Tacchi-Venturi. Mais on voit que ping-/seat ne donne pas la date de la composition d'une quelconque des parties du $n^{\circ} 996$, mais seulcment de l'exécution matérielle da manuscrit. On n'a même pas là un terminus ad quem, car ping-tseu pent à la rigueur correspondre à 1696. Le cachet de 思 默 Sseu-mo, qui se trouve au début da manuscrit, ne contredit pas ì cette dernière date; il en résulte en effet seulement que le manuscrit a appartenu au Jésuite chinois 陸㢈言 Lou Hi-yen, tseu Ssen-mo, qui a véeu de 1630 à 1704. Quant au «Rapport sur la vie du P. Ricci” par les PP. Pantoja et de Ursis qu'indique M. Courant ( $\left.n^{\circ} 1321\right)$, en disant qu'il jorte la date de 1586 qui serait sans doute à corriger en 1616 (ees mêmes indications ont passé dans Cordier, L'imprimerie sino-européenne, $\mathrm{n}^{\circ} 244$ ), le manuscrit donne très correctement et lisiblement la $44^{\prime}$ année Wan-li, c'est-à-dire 1616 (et non 1556), et ce n'est pas un rapport sur la viéda $\mathrm{P}$. Ricci, mais le mémorial présenté en 1616 par Pantoja et de Ursis pour se défendre contre les accusations de 泷漼 Chen Kio; en d'autres termes, c'est un exemplaire du 垶痛揭 Pien kie (sur lequel, ef. Cordier, L'imprimerie sino-européenne, $\mathrm{n}^{\circ}$ 184; Havret, Stc̀le chrétienze, II, 16-17). Par cet exemple, j’ai voulu seulement montrer que la bibliographie détaillée des cuvres chinoises du christianisme au $X V \backslash I^{c}$ siècle reste à faire, et que les éléments d'information ne font pas défaut. Mais lcur groupement et lenr discussion exigera beacoup de travail et un long temps.

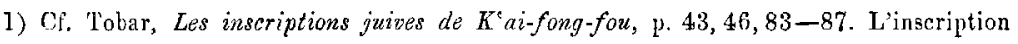
de 1663 énumère sept «familles», dont la famille Ngai, qui en 1653 Cournirent les fonds nécessaires pour reconstruire la synagogue. C'est plus de la moitié des c dis ou douze familles» dont Ngai Teien avait parlé à Ricei en 160 a (af. Tacchi-Venturi, I, 469). Il s'agit de «noms de famille» ou de «clans» différents, mais chacun deux étuit porté par un assez grand nombre d'individus. 
licence en Wan-li konei-yeou, c'est-à-dire en 1573, et ne dépassa pas les fonctions mandarivales de tche-hien, autrement dit de souspréfet $^{1}$ ). Il y a d'autant moins à douter que ce soit là l'iuformateur du P. Ricei qu'ayant passé la licence en 1573, Ngai T'ien derait bien avoir en 1605, comme le veut la lettre de Ricci, une soixantaine d'aunées. Par la lettre du P. Ricci, nous apprenons en outre que Ngai Trien reçut une fonction «daus une école de la ville de Ianceo». Il est pratiquement certain qu'il s'agit de la ville préfectorale de 揚州 Yang-tcheou au Kiang-sou, mais Ngai Tien eut lia une charge assez mince, et la Descriplion de $Y$ ang-lcheou est muette à son sujet.

J'ai rédigé cette note pour deux raisons. L'une est d'indiquer la voie par où on pent retrouver lans les textes chinois la grande majorité des personnages nomnés ou visés par les Commentaires de Ricei ou par ses Lettres ${ }^{2}$ ). Mais il m'a paru également intéressant de montrer, par un exemple concret, que, si le texte original des Commentaires permet de rectifier on de préciser en bien des endroits la version latine de Trigault, ces Commentaires n'en sont pas moins rédigés après coup, et qu'il est essentiel, chaque fois qu'on le peut, de les contrôler par les Lettres, qui sont, elles, un témoignage vraiment contemporain et d'une incomparable autorité.

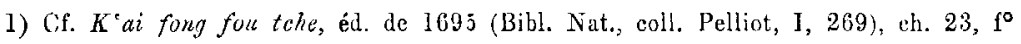

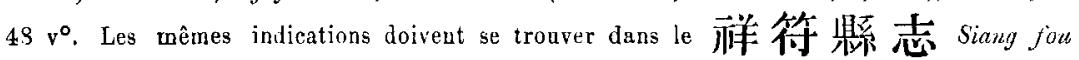
hien tche, mais l'cxcmplaire de la Bibliothèque Nationale (coll. Pelliot, II, 741; éd. de 1739) est très défectueux, et le passage en question y manque.

2) M. Vacea (dans Tacchi-Venturi, II, 143-144) a indiqué neuf équivalences (le nom

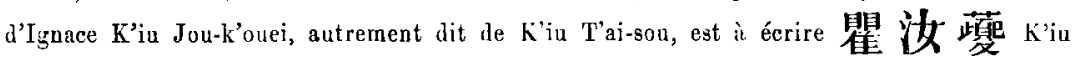
Jon-k'ouei; e'cst un oncle du ministre Thomas K'iu Che-sseu); il serait aisé de gnintupler ce chiffre.

Note additionnelle: Le Li Kicon-kong cité p. 38 est le père de 李秃芶 Li Yi-fen, éditeur de Courant, Catal, no ${ }^{\circ}$ 227. Ce Li Yi-len doit être le même que Lénnce Li, qui fut un des maîtres de chinois de $M^{\text {:r }}$ Maigrot. 